\title{
Relationship Between Rapid Skin Clearance and Quality of Life Benefit: Post Hoc Analysis of Japanese Patients with Moderate-to-Severe Psoriasis Treated with Ixekizumab (UNCOVER-J)
}

\author{
Masaru Honma - Zhihong Cai · Russel Burge - Baojin Zhu • \\ Sohiya Yotsukura $\cdot$ Hitoe Torisu-Itakura
}

Received: June 10, 2020 / Published online: September 10, 2020

(C) The Author(s) 2020

\begin{abstract}
Introduction: Ixekizumab has demonstrated rapid onset of action, high levels of skin clearance, and improvements in quality of life in patients with moderate-to-severe psoriasis, including plaque, erythrodermic, or generalized pustular psoriasis.
\end{abstract}

Methods: This was a post hoc analysis of UNCOVER-J, a phase 3, multicenter, single-arm, open-label study of ixekizumab for treatment of Japanese patients with psoriasis. The objective was to assess the proportion of patients who achieved Dermatology Life Quality Index

Digital Features To view digital features for this article go to https://doi.org/10.6084/m9.figshare.12834704.

Electronic supplementary material The online version of this article (https://doi.org/10.1007/s13555020-00441-4) contains supplementary material, which is available to authorized users.

\section{Honma}

Department of Dermatology, Asahikawa Medical

University, Asahikawa, Japan

Z. Cai $\cdot$ S. Yotsukura $(\bowtie) \cdot$ H. Torisu-Itakura

Eli Lilly Japan K.K., Kobe, Japan

e-mail: Yotsukura_sohiya@lilly.com

R. Burge · B. Zhu

Eli Lilly and Company, Indianapolis, IN, USA

R. Burge

Division of Pharmaceutical Sciences, University of

Cincinnati, Cincinnati, OH, USA
(DLQI) $(0,1)$ and Itch Numeric Rating Scale (NRS) (0) at weeks 4 and 12 according to Psoriasis Area and Severity Index (PASI) percentage improvement levels. All intent-to-treat patients with plaque, erythrodermic, or generalized pustular psoriasis were analyzed.

Results: A total of 91 patients were treated with ixekizumab and included in the analysis. Rapid improvements in PASI at weeks 4 and 12 were associated with improvements in DLQI $(0,1)$ response at week 4 and at week 12. Complete skin clearance (PASI 100) achieved either at week 4 or week 12 was associated with a higher Itch NRS (0) response at week 12.

Conclusions: Patients with rapid improvement in clinical symptoms of psoriasis had better patient outcomes than those with slower responses. These findings highlight the clinical importance of achieving a fast response in patients with psoriasis, which may lead to better treatment outcomes.

Trial Registration: ClinicalTrials.gov identifier, NCT01624233. 


\section{Graphic Abstract:}

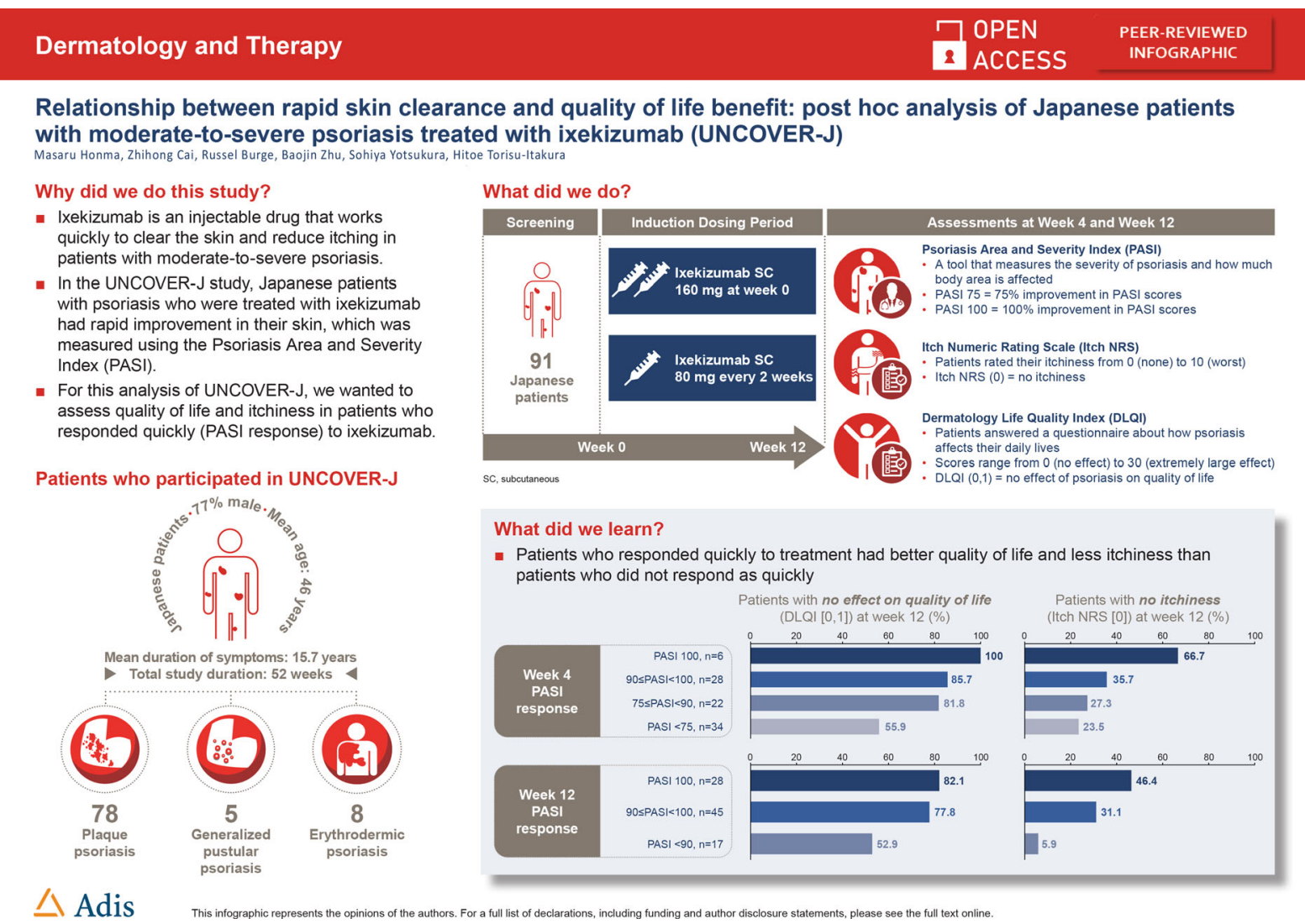

Keywords: Health-related quality of life; Ixekizumab; Japan; Psoriasis

\section{Key Summary Points}

\section{Why carry out this study?}

Ixekizumab has a rapid onset of action, high levels of skin clearance, and results in improvements in quality of life in patients with moderate-to-severe psoriasis, including plaque, erythrodermic, or generalized pustular psoriasis.

We hypothesized that rapid clinical responses to ixekizumab would result in better improvements in quality of life than slower responses.

\section{What was learned from the study?}

Patients who had rapid improvements in clinical symptoms of psoriasis (Psoriasis Area and Severity Index) had better improvements in quality of life (Dermatology Life Quality Index) and bothersome itch symptoms (Itch Numeric Rating Scale).

Treatments that provide rapid onset and complete or almost complete skin clearance may provide better quality-oflife outcomes for patients with moderateto-severe psoriasis. 


\section{INTRODUCTION}

Moderate-to-severe psoriasis is associated with bothersome symptoms and significantly decreased quality of life (QoL) [1-3]. Depending on local practice, patients may prefer systemic treatments that provide clear skin with a rapid onset [4]. Ixekizumab, a high-affinity monoclonal antibody that selectively targets interleukin-17A [5], has demonstrated rapid onset of action, high levels of skin clearance, and improvements in QoL $[6,7]$. Alleviation of itch in particular has been identified as one of the most important factors that contributes to improvements in QoL in ixekizumab-treated patients $[8,9]$.

The UNCOVER-J study of ixekizumab in Japanese patients with psoriasis [10] showed that most patients achieved a 75\% improvement in Psoriasis Area and Severity Index (PASI) score (PASI 75) after 12 weeks of treatment. For this post hoc analysis of UNCOVER-J, we hypothesized that rapid clinical responses to ixekizumab (PASI) would result in better improvements in QoL as measured by the Dermatology Life Quality Index (DLQI) and Itch Numeric Rating Scale (NRS) compared with slower responses.

\section{METHODS}

This was a post hoc analysis of the first 12 weeks of the 52-week, multicenter, single-arm, openlabel, phase 3 UNCOVER-J study of ixekizumab $\left(\right.$ Taltz $^{\circledR}$, Eli Lilly Japan K.K.) in Japanese patients with psoriasis (ClinicalTrials.gov, NCT01624233). Patients received a starting dose of $160 \mathrm{mg}$ ixekizumab, given as two $80 \mathrm{mg}$ subcutaneous injections at week 0 , then $80 \mathrm{mg}$ every 2 weeks up to and including week 12 , followed by $80 \mathrm{mg}$ every 4 weeks thereafter. Primary outcomes from UNCOVER-J have been reported previously [10]. The study was approved by local institution ethics committees (see Table S1, Electronic Supplementary Material) and complied with the Helsinki Declaration of 1964 and its later amendments. All patients provided written informed consent to participate in the study.
The objective of this analysis was to assess the proportion of patients who achieved DLQI $(0,1)$, indicating no effect of psoriasis on QoL [11], and Itch NRS (0), indicating no itch [12], at weeks 4 and 12 according to PASI percentage improvement levels. PASI is a standardized measure of psoriasis severity and involvement based on total body surface area affected and the degree of erythema, scaling, and induration [13]. Data from all intent-to-treat patients with plaque, erythrodermic, or generalized pustular psoriasis were included. Initially, patients were divided into four groups according to observed PASI responses at week 4: PASI percentage improvement $<75$ (PASI $<75$ ); $\geq 75$ to $<90$ PASI percentage improvement $(75 \leq$ PASI $<$ $90) ; \geq 90$ to $<100$ PASI percentage improvement $(90 \leq$ PASI $<100)$; and PASI percentage improvement 100 (PASI 100). Because most patients treated with ixekizumab achieved PASI $\geq 75$ at week 12 , PASI improvement at week 12 was categorized as PASI $<90,90 \leq$ PASI $<100$, and PASI 100 . The association between proportions of patients who achieved DLQI $(0,1)$ and Itch NRS $(0)$ at weeks 4 and 12 by PASI responses at weeks 4 and 12 were assessed using a chi-square test. Receiver operating characteristic (ROC) curves were used to determine the optimum week 4 PASI percentage improvement that could predict DLQI $(0,1)$ and Itch NRS (0) responses at week 12 . The area under the curve (AUC) of the ROC curves was used to assess the predictability of DLQI $(0,1)$ and Itch NRS (0) responses. The Youden index was used to select the optimum sensitivity and specificity from the ROC curves. All analyses and descriptive statistics were conducted using SAS version 9.4, SAS Institute, Cary, NC.

\section{RESULTS}

A total of 91 patients were included in the analyses (Table 1). The mean age of patients was 46 years, $77 \%$ were male, $86 \%$ had plaque psoriasis, and the average duration of symptoms was 15.7 years. 
Table 1 Baseline demographics and disease characteristics

\begin{tabular}{ll}
\hline Characteristics & $\boldsymbol{N}=\mathbf{9 1}$ \\
\hline Age, years & $46.2(11.9)$ \\
Sex, male, $n$ (\%) & $70(76.9)$ \\
Weight, kg & $72.5(16.6)$ \\
Plaque psoriasis, $n$ (\%) & $78(85.7)$ \\
Erythrodermic psoriasis, $n$ (\%) & $8(8.8)$ \\
Generalized pustular psoriasis, $n$ (\%) & $5(5.5)$ \\
Duration of psoriasis symptoms, years & $15.7(10.3)$ \\
PASI & $27.2(10.5)$ \\
DLQI score & $10.8(6.5)$ \\
Itch NRS score & $6.1(2.5)$ \\
\hline
\end{tabular}

Data are mean (standard deviation) unless stated otherwise DLQI Dermatology Life Quality Index, NRS Numeric Rating Scale, PASI Psoriasis Area and Severity Index

\section{DLQI $(0,1)$ Response}

An association between PASI improvement at week 4 and DLQI $(0,1)$ responses was observed at week 4 and at week 12 (Fig. 1a, b). At week 4 , the DLQI $(0,1)$ response rate was highest for patients with week 4 PASI 100 (83.3\%), followed by $90 \leq$ PASI $<100 \quad(58.6 \%), \quad 75 \leq$ PASI $<90$ (45.5\%), and PASI $<75$ (32.4\%). At week 12 , the DLQI $(0,1)$ response rate had increased in all week 4 PASI groups and the DLQI $(0,1)$ response rate was highest for patients with week 4 PASI 100 (100\%), followed by $90 \leq$ PASI $<100$ $(85.7 \%), 75 \leq$ PASI $<90(81.8 \%)$, and PASI $<75$ $(55.9 \%)$. Similar increases were observed for the association between week 12 PASI improvement and DLQI $(0,1)$ response rate at week 12 (Fig. 1c).

\section{Itch NRS (0) Response}

Higher PASI improvement levels, especially with week 4 PASI 90 or greater, were associated with greater numerical improvement in Itch NRS (0) responses at weeks 4 and 12 (Fig. 2a, b). At week 12, the Itch NRS (0) response rate had increased in all week 4 PASI groups and the Itch NRS (0) response rate was highest for patients with week 4 PASI 100 (66.7\%), followed by $90 \leq$ PASI $<100 \quad(35.7 \%), \quad 75 \leq$ PASI $<90$ $(27.3 \%)$, and PASI $<75$ (23.5\%). An association between week 12 PASI improvement and Itch NRS (0) responses at week 12 was evident, with a clear trend toward increasing improvement in Itch NRS (0) with increasing levels of improvement in PASI (Fig. 2c).

\section{Predictive Value of PASI Percentage Improvement at Week 4}

The predictability of a DLQI $(0,1)$ response was $70.3 \%$ and the analysis showed that an $82 \%$ improvement in PASI at week 4 was the optimum threshold for predicting a DLQI $(0,1)$
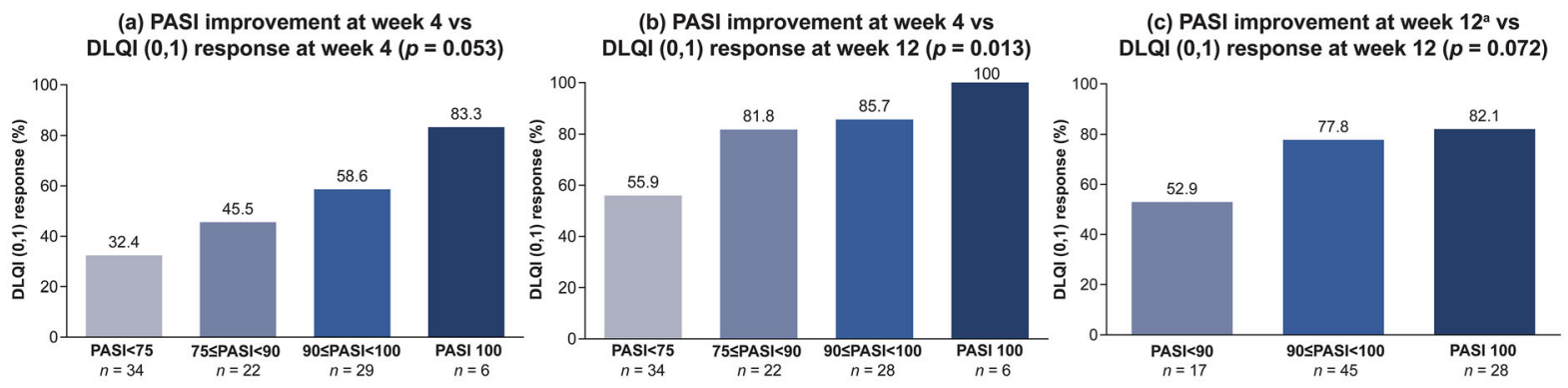

Fig. 1 Association between Psoriasis Area and Severity Index (PASI) improvement and Dermatology Life Quality Index (DLQI) $(0,1)$ response rates in patients treated with ixekizumab for 4 and 12 weeks. DLQI $(0,1)$ represents an outcome of no effect of skin condition on patient's quality

of life; PASI $75 / 90 / 100=75 \% / 90 \% / 100 \%$ reduction in PASI. ${ }^{a}$ At week 12, the groups for PASI $<75$ and $75 \leq$ PASI $<90$ were combined because most patients achieved PASI $\geq 75$ at week 12 . $p$, chi-square test 

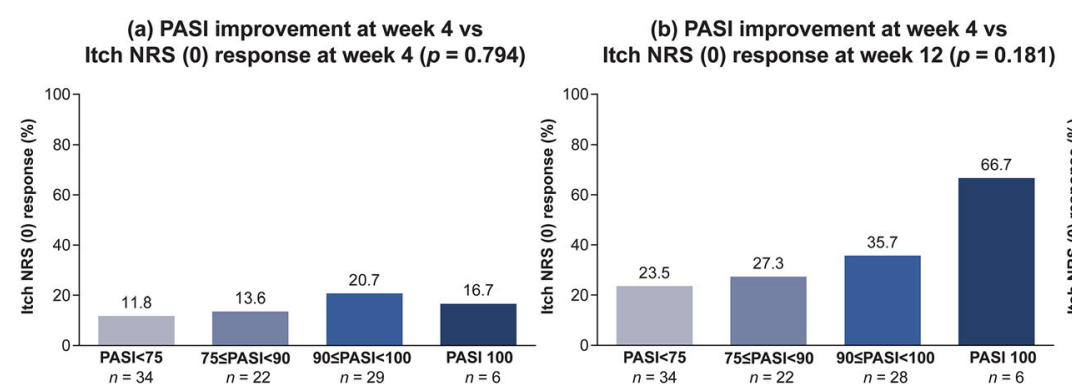

(c) PASI improvement at week $12^{\mathrm{a}}$ vs Itch NRS $(0)$ response at week $12(p=0.017)$

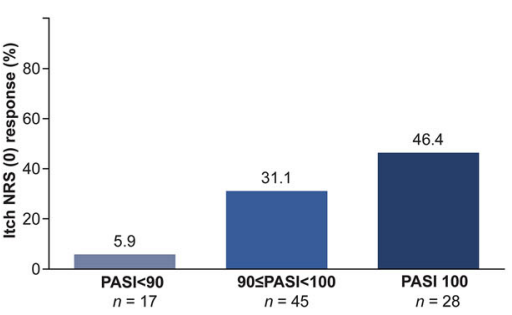

Fig. 2 Association between Psoriasis Area and Severity Index (PASI) improvement and Itch Numeric Rating Scale (NRS) (0) response rates in patients treated with ixekizumab for 4 and 12 weeks. Itch NRS (0) represents complete resolution of itch; PASI 75/90/100 $=75 \%$ /

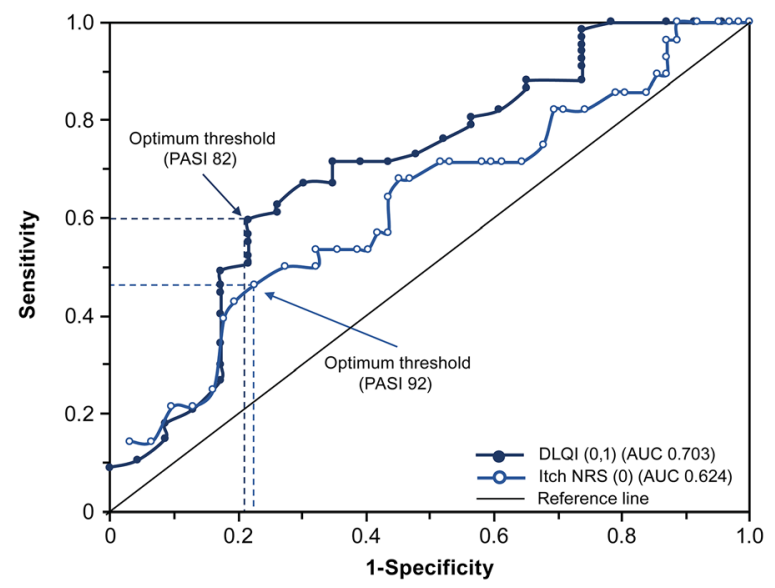

Fig. 3 Receiver operating characteristic curves for the week 4 Psoriasis Area and Severity Index (PASI) percentage improvement for predicting Dermatology Life Quality Index (DLQI) $(0,1)$ and Itch Numeric Rating Scale (NRS) (0) response rates in patients treated with ixekizumab for 12 weeks. DLQI $(0,1)$ represents an outcome of no effect of skin condition on patient's quality of life and Itch NRS (0) represents complete resolution of itch. AUC area under the curve

response at week 12 , with a sensitivity of $59.7 \%$ and a specificity of $78.3 \%$ (Fig. 3). The predictability of an Itch NRS (0) response was $62.4 \%$ and a $92 \%$ improvement in PASI at week 4 was the optimum threshold for predicting an Itch NRS (0) response at week 12, with a sensitivity of $46.4 \%$ and a specificity of $77.4 \%$.
$90 \% / 100 \%$ reduction in PASI. ${ }^{a}$ At week 12 , the groups for PASI $<75$ and $75 \leq$ PASI $<90$ were combined because most patients achieved PASI $\geq 75$ at week 12. $p$, chi-square test

\section{DISCUSSION}

This analysis was designed to quantify differential health outcome improvements based on the observed proportion of patients who achieved DLQI $(0,1)$ and Itch NRS $(0)$ at weeks 4 and 12 according to PASI improvement levels. Baseline PASI in the UNCOVER-J study population was 27.2, which was higher than observed in the global populations of the UNCOVER-1, 2 , and -3 trials of ixekizumab (19-21) [7]. The higher PASI score at baseline in UNCOVER-J is likely because this study included patients with plaque psoriasis and the more severe types of psoriasis, erythrodermic and generalized pustular psoriasis, which can be more challenging to treat. Therefore, achieving PASI 100 in UNCOVER-J is especially difficult given the higher baseline PASI levels in this population. Patients who had rapid improvement in clinical symptoms of psoriasis (PASI) had better patient outcomes when compared to patients with slower responses. In addition, the rapid improvement in PASI at week 4 was associated with improvement in QoL (DLQI) and bothersome symptom (Itch NRS) outcomes at week 12 in our cohort of Japanese patients with moderate-to-severe plaque, erythrodermic, or generalized pustular psoriasis. The ROC curve analysis indicated that week 4 PASI improvement showed predictability for DLQI $(0,1)$ and Itch NRS (0) responses at week 12 and that week 4 PASI improvement could predict DLQI 
$(0,1)$ responses better than Itch NRS (0) responses.

Findings from a study of 3066 patients from the German Psoriasis Registry who had moderate-to-severe psoriasis at the start of systematic treatment showed that more than 90\% nominated "skin healing", "quick skin improvement", "confidence in therapy", and "control over the disease" as quite or very important treatment goals [4]. Together with the current study, these findings suggest that treatments that provide high levels of improvement in skin healing during the early stages of therapy may be of benefit for many patients with moderateto-severe psoriasis not only in terms of clinical outcomes and improvements in QoL but also in terms of patient preferences for a sense of control over their disease and confidence with therapy.

The main strengths of the study are the multicenter design and the inclusion of patients with severe forms of psoriasis (erythrodermic and generalized pustular psoriasis). Ixekizumab has shown efficacy in this difficult-to-treat population. However, this study is limited by the single-arm, open-label study design and the small sample size of Japanese patients. As a result, study findings may not be generalizable to other populations.

\section{CONCLUSION}

Overall, these findings highlight the clinical importance of a fast response to treatment in patients with psoriasis. Treatments that provide rapid onset and complete skin clearance may provide better outcomes during the early stages of treatment for patients with moderate-to-severe psoriasis.

\section{ACKNOWLEDGEMENTS}

The authors would like to thank all study participants and all members of the Japanese Ixekizumab Study Group who enrolled patients with erythrodermic psoriasis or generalized pustular psoriasis: Atsuyuki Igarashi of NTT Medical Center Tokyo, Chika Ohata of Kurume
University Hospital, Kazuhiko Takehara of Kanazawa University Hospital, Yoshinori Umezawa of The Jikei University Hospital, and Keiichi Yamanaka of Mie University Graduate School of Medicine.

Funding. This study and the Rapid Service Fee were funded by Eli Lilly Japan K.K., the manufacturer/licensee of Taltz ${ }^{\circledR}$. Eli Lilly Japan K.K. was involved in the study design, data collection, data analysis, and preparation of the manuscript.

Authorship. All named authors meet the International Committee of Medical Journal Editors (ICMJE) criteria for authorship for this article, take responsibility for the integrity of the work as a whole, and have given their approval for this version to be published. M. Honma was an investigator in the study and involved in data collection and R. Burge, B. Zhu, and Z. Cai were involved in the study design.

Medical Writing Assistance. Medical writing assistance was provided by Serina Stretton, PhD, CMPP, of ProScribe-Envision Pharma Group, and was funded by Eli Lilly Japan K.K. ProScribe's services complied with international guidelines for Good Publication Practice (GPP3).

Prior Presentation. Data from this manuscript have been previously reported, in part, at the 24th World Congress of Dermatology; Milan, Italy; June 10-15, 2019.

Disclosures. Masaru Honma has no conflicts of interest to declare. Russel Burge and Baojin Zhu are employees of Eli Lilly and Company. Zhihong Cai, Sohiya Yotsukura, and Hitoe Torisu-Itakura are employees of Eli Lilly Japan K.K. Russel Burge, Baojin Zhu, Zhihong Cai, and Hitoe Torisu-Itakura are minor shareholders of Eli Lilly and Company.

Compliance with Ethics Guidelines. The study was approved by local institution ethics committees (see Table S1, Electronic Supplementary Material) and complied with the Helsinki Declaration of 1964 and its later 
amendments. All patients provided written informed consent to participate in the study.

Data Availability. Lilly provides access to all individual participant data collected during the trial, after anonymization, with the exception of pharmacokinetic or genetic data. Data are available to request 6 months after the indication studied has been approved in the USA and EU and after primary publication acceptance, whichever is later. No expiration date of data requests is currently set once data are made available. Access is provided after a proposal has been approved by an independent review committee identified for this purpose and after receipt of a signed data sharing agreement. Data and documents, including the study protocol, statistical analysis plan, clinical study report, blank or annotated case report forms, will be provided in a secure data sharing environment. For details on submitting a request, see the instructions provided at http:// www.vivli.org.

Open Access. This article is licensed under a Creative Commons Attribution-NonCommercial 4.0 International License, which permits any non-commercial use, sharing, adaptation, distribution and reproduction in any medium or format, as long as you give appropriate credit to the original author(s) and the source, provide a link to the Creative Commons licence, and indicate if changes were made. The images or other third party material in this article are included in the article's Creative Commons licence, unless indicated otherwise in a credit line to the material. If material is not included in the article's Creative Commons licence and your intended use is not permitted by statutory regulation or exceeds the permitted use, you will need to obtain permission directly from the copyright holder. To view a copy of this licence, visit http://creativecommons.org/licenses/by$\mathrm{nc} / 4.0 /$.

\section{REFERENCES}

1. Edson-Heredia E, Zhu B, Guo J, Maeda-Chubachi T, Lebwohl M. Disease burden and quality of life in psoriasis patients with and without comorbid psoriatic arthritis: results from National Psoriasis Foundation panel surveys. Cutis. 2015;95(3):173-8.

2. Takahashi H, Satoh K, Takagi A, Iizuka H. Cost-efficacy and pharmacoeconomics of psoriatic patients in Japan: analysis from a single outpatient clinic. J Dermatol. 2019;46(6):478-81.

3. Mabuchi T, Yamaoka H, Kojima T, Ikoma N, Akasaka E, Ozawa A. Psoriasis affects patient's quality of life more seriously in female than in male in Japan. Tokai J Exp Clin Med. 2012;37(3):84-8.

4. Blome C, Gosau R, Radtke MA, et al. Patient-relevant treatment goals in psoriasis. Arch Dermatol Res. 2016;308(2):69-78.

5. Liu L, Lu J, Allan BW, et al. Generation and characterization of ixekizumab, a humanized monoclonal antibody that neutralizes interleukin-17A. J Inflamm Res. 2016;9:39-50.

6. Griffiths CE, Reich K, Lebwohl M, et al. Comparison of ixekizumab with etanercept or placebo in moderate-to-severe psoriasis (UNCOVER-2 and UNCOVER-3): results from two phase 3 randomised trials. Lancet. 2015;386(9993):541-51.

7. Gordon KB, Blauvelt A, Papp KA, et al. Phase 3 trials of ixekizumab in moderate-to-severe plaque psoriasis. N Engl J Med. 2016;375(4):345-56.

8. Yosipovitch G, Dong Y, Burge R, Zhu B, Shrom D, Kimball AB. Assessing the impact of improvements in PASI and itch scores on patients' quality of life in the treatment of psoriasis. Acta Derm Venereol. 2019;99(11):1031-2.

9. Zhu B, Edson-Heredia E, Guo J, Maeda-Chubachi T, Shen W, Kimball AB. Itching is a significant problem and a mediator between disease severity and quality of life for patients with psoriasis: results from a randomized controlled trial. Br J Dermatol. 2014;171(5):1215-9.

10. Saeki H, Nakagawa H, Ishii T, et al. Efficacy and safety of open-label ixekizumab treatment in Japanese patients with moderate-to-severe plaque psoriasis, erythrodermic psoriasis and generalized pustular psoriasis. J Eur Acad Dermatol Venereol. 2015;29(6):1148-55.

11. Finlay AY, Khan GK. Dermatology Life Quality Index (DLQI) - a simple practical measure for routine clinical use. Clin Exp Dermatol. 1994;19(3): 210-6. 
12. Phan NQ, Blome C, Fritz F, et al. Assessment of pruritus intensity: prospective study on validity and reliability of the visual analogue scale, numerical rating scale and verbal rating scale in 471 patients with chronic pruritus. Acta Derm Venereol. 2012;92(5):502-7.
13. Garduno J, Bhosle MJ, Balkrishnan R, Feldman SR. Measures used in specifying psoriasis lesion(s), global disease and quality of life: a systematic review. J Dermatol Treat. 2007;18(4):223-42. 